\title{
Artificial Intelligence in Automated Sorting in Trash Recycling
}

\author{
Bernardo S. Costa ${ }^{1}$, Aiko C. S. Bernardes ${ }^{1}$, Julia V. A. Pereira ${ }^{1}$, \\ Vitoria H. Zampa ${ }^{1}$, Vitoria A. Pereira ${ }^{1}$, Guilherme F. Matos ${ }^{3}$ \\ Eduardo A. Soares ${ }^{2}$, Claiton L. Soares ${ }^{3}$, Alexandre F. Silva ${ }^{1}$ \\ ${ }^{1}$ Department of Computer - Federal Institute of South of Minas Gerais (IFSuldeMinas) \\ CEP 37.550-000 - Pouso Alegre - MG - Brazil \\ ${ }^{2}$ Department of Computer - University of São Carlos - SP - Brazil \\ ${ }^{3}$ Departament of Computer - Federal Institute of Triângulo Mineiro - MG - Brazil \\ \{bernardocosta122, aikocamillle, jujupereira1107, vitysshelena, \\ vitoriaaparecida.pereira,GuilhermeFMatos.ptu, edu.soares999\}@gmail.com, \\ claitonsoares@iftm.edu.br, alexandre.silva@ifsuldeminas.edu.br
}

\begin{abstract}
A computer vision approach to classify garbage into recycling categories could be an efficient way to process waste. This project aims to take garbage waste images and classify them into four classes: glass, paper, metal and, plastic. We use a garbage image database that contains around 400 images for each class. The models used in the experiments are Pre-trained VGG-16 (VGG16), AlexNet, Support Vector Machine (SVM), K-Nearest Neighbor (KNN) and, Random Forest $(R F)$. Experiments showed that our models reached accuracy around $93 \%$.
\end{abstract}

\section{Introduction}

Knowing that a good part of the generated garbage in large cities are recyclables, it is need to know and apply reuse methods that could bring benefits or, at least reduce environmental problems. The existence of techniques or models that help people to sort garbage has become essential in the correct dispose of those materials. Although there are different types of recycling categories, people still can be confused or do not properly recognize about how to determine the correct trash bin can to dispose of each garbage.

In order to minimize the impact caused by the incorrect dispose of garbage, more specifically domestic (i.e., paper, plastic, glass and trash), we proposed to use an automated system based on neural network techniques aiming at the correct separation of waste in recycling categories. Ways which humans have managed solid waste over the centuries still based on the original strategy of just eliminate them. Population growth has been the main factor for the increasing production of those garbages. Therefore, it should be reduced on a personal basis to maintain the balance at which the waste is managed [Islam et al. 2012].

Waste management and efficient sorting of them have been considered as an important role for ecologically sustainable development worldwide. It is essential for the society to reduce waste accumulation by recycling and re-using disposed of products. Efficient selective sorting is often implemented to improve recycling and reduce the environment impact [Glouche and Couderc 2013]. This problem should be specially treated 
in the developing countries, where waste management is a severe problem for their urbanization and economic development [Arebey et al. 2011].

Our motivation is concerning to find an automatic method for sorting waste aiming help reduce waste and the pollution. This will not only have positive environmental effects but also beneficial economic effects. In addition, our system has a great community appeal by adding the value of knowledge and the social stimulus in the separation and disposal of garbage. So, we investigate the different types of neural networks (NN) to classify the garbage waste images into four classes: glass, paper, metal, and plastic. Then, we will address the following open research questions: (1) are NN techniques efficient for learning good feature representations from images to tackle recycling sort?; and, (2) how much improvements can be achieved by NN techniques when compared with others works?

Novelties of this paper includes, the proposition of a system to assist the user to determine in which trash can discard waste, based in an image of the material gathered from digital camera, therefore, it requires less human contact. The proposed work select the best trash bin considering each type of waste, in a similar way as in Yang M. et al. [Yang and Thung ]. Also, we go beyond of Yang M. et al. [Yang and Thung] by testing different neural networks.

The rest of the paper is organized as following. Section 2 introduces the related work. Section 3 presents the experimental methodology, which includes the image database used and, the models and methods. Section 4 introduces the experimental results. Conclusions are detailed in Section 5 .

\section{Related Work}

Garbage has become a major problem worldwide due to uncontrolled disposal of household waste from citizen's home and industries without an effective and efficient waste management program that can result in health risks and a negative impact on the environment [Islam et al. 2012].

A waste management with efficient classification play an important role in ecologically sustainable development by ensuring that waste is properly disposed of. Efficient selective collection is often implemented to improve recycling and reduce environmental impact [Glouche and Couderc 2013], especially in developing countries where waste management is a serious problem for economic development [Arebey et al. 2011].

Over the years, many works have been implemented with the aim of minimizing the impact of the waste uncontrolled disposal of. Technologies such as Radio Frequency Identification (RFID) and Sensor Network (SN) have been used to provide a new way to optimize waste management systems [Glouche and Couderc 2013]. Some authors have used RFID technology to identify and track selective collection by storing owner data and information about their bins.

Glouche Y. et al. [Glouche and Couderc 2013] proposed a method to improve the quality of selective collection by tracking the waste stream of a city, where each waste is detected from information stored on an RFID tag (associated with waste) and during the waste processing step RFID tags are read to provide some relevant information. In Arebey, M. et al. [Arebey et al. 2011] was proposed to estimate household waste volume 
based on image analysis of the contents of the open container lid from RFID tags, such as, the label would be used to associate each bin to the address of the house that owns it. In Abdoli, S. [ABDOLI 2009], the authors discussed applications of RFID technology in self-management of products, with emphasis on municipal solid waste management and its environmental implications, also by identifying each bin from an RFID tag. In Chowdhury, B. et al. [Chowdhury and Chowdhury 2007], the authors proposed a RFID sensor (to store the weight of the bin and to identify the owner of each bin) that measures in each collection operation, the weight of the waste bin discarded in the garbage collection truck. In Swedberg, C. [SWedberg 2008] the authors tracked the consumers by identifying them using an RFID tag associated with their recycle bin. Consumers are also financially rewarded based on the weight of their recycling packages or how much they recycled. Parlikad, A.K. et al. [Parlikad and McFarlane 2007] used RFID tags in their products to provide information to recycling service providers aiming to help in deciding on the appropriate treatment of that product. In Thomas, V.M. [Thomas 2008], the author discussed the use of RFID in waste management and a system of discounts and fees to stimulate responsible behavior of the population in the screening process. At this point, the works presented are based on the problem of identifying, tracking and to analyze the discarded garbage in order to stimulate the responsible behavior of the population for selective collection. However, none of these studies aimed to assist the population in the correct disposal of garbage.

Aiming to help the consumers properly discard waste, Sinha et al. [Sinha and Couderc 2012] developed a method capable of selecting the best recycling container considering the waste discarded by the user. They presented a model using Ontology Web Language (OWL) to sort the smart waste items for better recycling of materials. They use the OWL ontology to represent information about the amount of valuable recyclable materials contained in each waste. Yang M. et al. [Yang and Thung] proposed an approach to classifying garbage into six different recycling categories (i.e., metal, paper, glass, plastic, trash, and cardboard) by using a support vector machine (SVM), with scale-invariant feature transform (SIFT) features, and a convolutional neural network (CNN). Their approaches achieved an accuracy rate of $63 \%$ and $22 \%$ for the trained SVM and the CNN, respectively. In a similar work, Oluwasanya Awe et al. [Awe et al. 2017] proposed a method to categorize the different wastes into three categories (eg., landfill, recycling and, paper) of an image of a jumbled waste by using faster Region-based Convolutional Neural Networks (Faster R-CNN) technique to get region proposals and classify objects. Our work is similar to the work of Yang et al. [Yang and Thung], with the difference that we trained the images with others NN techniques, such as, Support Vector Machine (SVM), Pre-trained VGG-16 (VGG16), AlexNet, K-Nearest Neighbor (KNN) and, random forest (RF).

\section{Experimental Methodology}

Finding a method that identifying the trash where a particular waste should be disposed of is important for properly collective selection. So, we test some neural network approaches in order to verify which one reaches the best performance in determining in which trash bin can discard waste.

We use the image database created by Yang et al. [Yang and Thung] containing images of recycled objects across six classes with about 400-500 images each, totaling 
about 2,400 images. According the authors, the data acquisition process involved using a white poster-board as a background and the lighting and pose for each photo is not the same, which introduces variation in the database. Figure 1 show samples of images of recycled objects around of six classes.

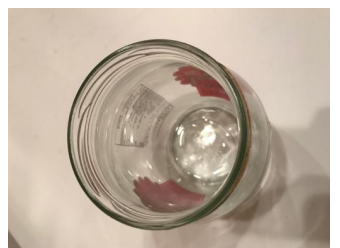

(a) Glass

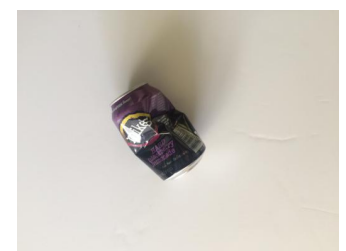

(b) Metal

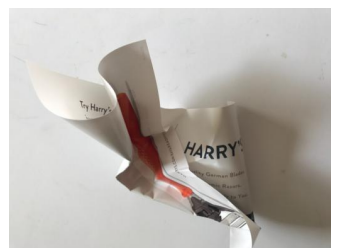

(c) Paper

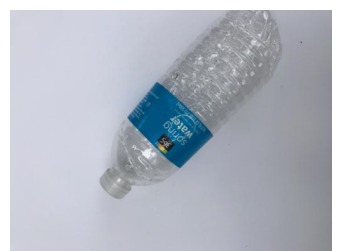

(d) Plastic

Figure 1. Some samples of images of recycled objects of database found in |Yang and Thung ]

To estimate the performance of each neural network, we calculate the Pearson correlation coefficient (PCC) and the Spearman Rank Order Correlation Coefficient (SCC). The models used in the experiments were VGG-16 model (VGG16), AlexNet, Support Vector Machine (SVM), K-Nearest Neighbor (KNN) and, Random Forest (RF).

The VGG16 is a pre-trained Convolutional Neural Network (CNN) that has been trained on approximately 1.2 million images from the ImageNet Dataset by K. Simonyan et al. [Simonyan and Zisserman 2014]. The model has 16 layers and can classify images into 1000 object categories. Also, VGG16 came up with significantly more accurate ConvNet architectures, which not only achieved the state-of-the-art accuracy on ImageNet Large-Scale Visual Recognition Challenge (ILSVRC) classification and localisation tasks, but are also applicable to other image recognition datasets, where they achieve excellent performance even when used as a part of a relatively simple pipelines (e.g. deep features classified by a linear SVM without fine-tuning) [Simonyan and Zisserman 2014].

VGG-16 and AlexNet are often used in photo classification, as a large fraction of examples in ImageNet are composed of photos. Both reaches a low error when trained over the million of images contained in ImageNet. ImageNet is a large collection of hierarchical labeled images that is used in the ImageNet Challenge [Russakovsky et al. 2015]. AlexNet was the first famous convolutional neural network (CNN) that included repeating a few convolutional layers each followed by max-poolings [Krizhevsky et al. 2012].

Also, we use three different classification algorithms: SVM, KNN and RF. SVMs is a supervised machine learning algorithm that can be employed for both classification and regression purposes. SVMs are based on the idea of finding a hyperplane that best divides a dataset into two classes (support vectors). Support vectors are the data points nearest to the hyperplane (as a simple example, for a classification task with only two features the hyperplane can be considered as a line that linearly separates and classifies a set of data), the points of a data set that, if removed, would alter the position of the dividing hyperplane [Cortes and Vapnik 1995]. KNN is one of the first supervised classifier. The simpler version of the K-nearest neighbor classifier algorithms is to predict the target label by finding the nearest neighbor class. The closest class will be identified using the distance measures like Euclidean distance [Altman 1992]. RF is a supervised 
classification algorithm and, in general, the more trees in the forest the more robust the forest seems to be. In the same way in the random forest classifier, the higher the number of trees in the forest gives the high accuracy results [Ho 1995].

\section{Experimental Results}

Computational simulations were performed to assess the usefulness and accuracy of those classifiers. Table 1 summarizes the results obtained by VGG-16, AlexNet, KNN, SVM and the RF classifiers model for automated sorting in trash recycling.

Table 1. Average correlation among classifiers

\begin{tabular}{c|c}
\hline \hline Classifiers & Accuracy \\
\hline VGG-16 & $\mathbf{9 3 . 0 \%}$ \\
AlexNet & $91.0 \%$ \\
KNN & $88.0 \%$ \\
SVM & $80.0 \%$ \\
RF & $85.0 \%$ \\
\hline \hline
\end{tabular}

We can notice that both CNN approaches performed better than the traditional machine learning ones. However, CNN require a substantial amount of time to train, and tune to achieve optimal performance. To improve $\mathrm{CNN}$ results, more data could be used. Furthermore, one could maximize the data through augmentation. Along with that, more thorough hyperparameter search could be performed. Figure 2 shows the confusion matrix for the best scenario of automated sorting in trash recycling classification (i.e., VGG-16 approach). We can to notice that glass is the most difficult material to classify. We suppose this results taking into account the texture of glass, once that the VGG-16 classifier may confound it with plastic or metal.

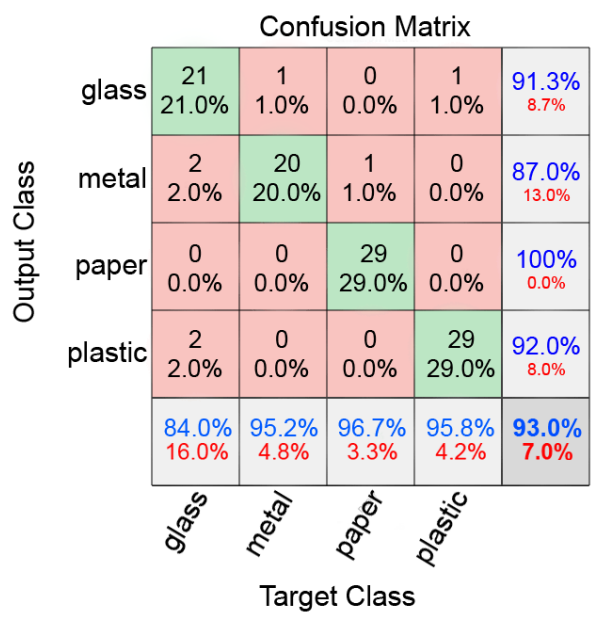

Figure 2. Confusion Matrix for VGG-16 classifier.

We perform a balanced one-way Analysis of Variance (ANOVA) to compare the classifiers methods [Antonisamy et al. 2017] considering the null hypothesis as that the 
mean accuracy of the methods is essentially the same and, a cutoff value $p<0.05$ suggests that the accuracy, at least, one of the algorithms is significantly different from each other. As resulted, a $p=0.0012$ was obtained indicating that the mean accuracy of the algorithms are not all the same; the null hypothesis was rejected. The Tukey Honestly Significant Difference (HSD) test [Antonisamy et al. 2017] was performed to compare pairs of algorithms, although this test is optimal for balanced one-way ANOVA and for similar procedures with equal sample sizes. Table 2 shows the results of the Tuckey HSD test for a $95 \%$ confidence interval (CI) for the difference of the means.

Table 2. Average correlation among classifiers

\begin{tabular}{cc|ccc}
\hline \hline Method 1 & Method 2 & CI Lower bound & Diff of Means & CI Upper bound \\
\hline VGG-16 & AlexNet & 0.0180 & 0.0300 & 0.0420 \\
VGG-16 & KNN & 0.0380 & 0.0500 & 0.0619 \\
VGG-16 & SVM & 0.0128 & 0.1400 & 0.1519 \\
VGG-16 & RF & 0.0680 & 0.0800 & 0.0919 \\
\hline \hline
\end{tabular}

The results show that the difference between the methods is significant at the 0.05 level. As shown in the Figure 3, VGG-16 is statistically superior (diamond symbol on top right in Fig. 3) compared to other classifiers models in terms of prediction accuracy.

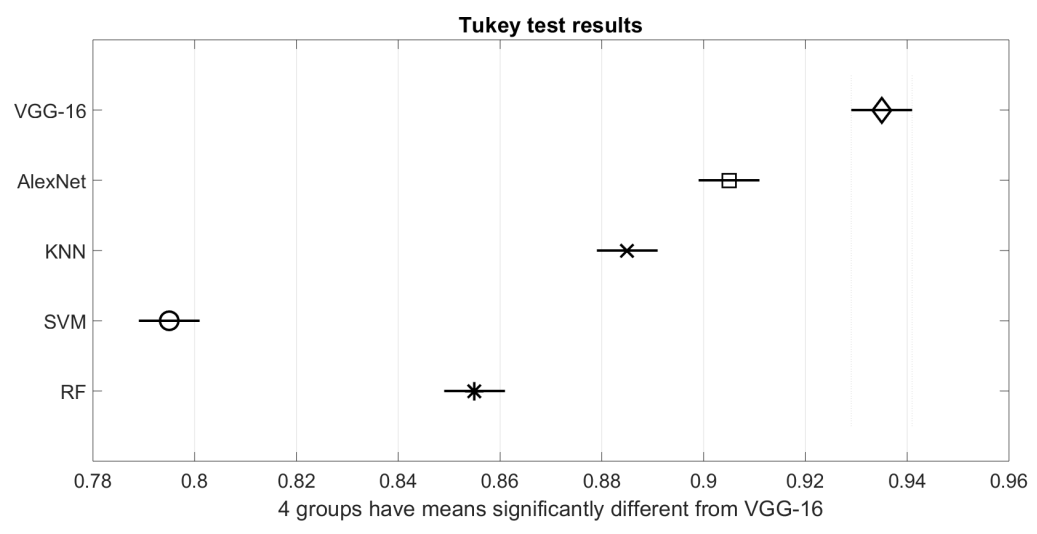

Figure 3. Tukey Test Results.

Since the difference in accuracy between VGG-16 and the others classifiers models is statistically significant, we can conclude that the method used to classify the materials to be dispose of in the trash recycling is efficient for the problem approached. In general, experiments on automated sorting trash recycling have shown that CNN are an efficient approach for this task. Classification accuracies were higher than those produced by traditional machine learning approaches. However, The number of algebraic calculations for $\mathrm{CNN}$ is larger and therefore, the method is slower than the traditional ones. Moreover, CNN can improve their accuracies using some techniques, such as, augmentation, fine tuning, etc.

\section{Conclusion}

In order to minimize the impact caused by the incorrect dispose of garbage, more specifically domestic (i.e., paper, plastic, glass and organic), we propose an automated system 
based on deep learning approach and traditional techniques by aiming the correct separation of waste in recycling categories. Four different trash categories were considered: glass, metal, paper and, plastic. Results have shown that VGG-16 methods is an efficient approach for this problem, reaching $93 \%$ of accuracy in its best scenario. However, CNN approaches tends to be more computational expensive than the traditional techniques, requiring better computational resources. Also, the accuracy in CNN approaches can be improved trough some techniques as augmentation, and fine tuning, which will be investigated in future work. Moreover, if more data is available, CNN tends to produce better results. Future work will also investigate, non-black-box approaches such as deep fuzzy rule-based approaches, which provides human interpretable rule.

\section{ACKNOWLEDGMENT}

This work is supported by Federal Institute of Education, Science and Technology of South of Minas Gerais - IFSULDEMINAS.

\section{References}

[ABDOLI 2009] ABDOLI, S. (2009). Rfid application in municipal solid waste management system.

[Altman 1992] Altman, N. S. (1992). An introduction to kernel and nearest-neighbor nonparametric regression. The American Statistician, 46(3):175-185.

[Antonisamy et al. 2017] Antonisamy, B., Premkumar, P. S., and Christopher, S. (2017). Principles and Practice of Biostatistics-E-book. Elsevier Health Sciences.

[Arebey et al. 2011] Arebey, M., Hannan, M., Basri, H., Begum, R. A., and Abdullah, H. (2011). Integrated technologies for solid waste bin monitoring system. Environmental monitoring and assessment, 177(1-4):399-408.

[Awe et al. 2017] Awe, O., Mengistu, R., and Sreedhar, V. (2017). Smart trash net: Waste localization and classification.

[Chowdhury and Chowdhury 2007] Chowdhury, B. and Chowdhury, M. U. (2007). Rfidbased real-time smart waste management system. In Telecommunication Networks and Applications Conference, 2007. ATNAC 2007. Australasian, pages 175-180. IEEE.

[Cortes and Vapnik 1995] Cortes, C. and Vapnik, V. (1995). Support-vector networks. Machine learning, 20(3):273-297.

[Glouche and Couderc 2013] Glouche, Y. and Couderc, P. (2013). A smart waste management with self-describing objects. In The Second International Conference on Smart Systems, Devices and Technologies (SMART'13).

[Ho 1995] Ho, T. K. (1995). Random decision forests. In Document analysis and recognition, 1995., proceedings of the third international conference on, volume 1, pages 278-282. IEEE.

[Islam et al. 2012] Islam, M. S., Arebey, M., Hannan, M., and Basri, H. (2012). Overview for solid waste bin monitoring and collection system. In Innovation Management and Technology Research (ICIMTR), 2012 International Conference on, pages 258-262. IEEE. 
[Krizhevsky et al. 2012] Krizhevsky, A., Sutskever, I., and Hinton, G. E. (2012). Imagenet classification with deep convolutional neural networks. In Advances in neural information processing systems, pages 1097-1105.

[Parlikad and McFarlane 2007] Parlikad, A. K. and McFarlane, D. (2007). Rfid-based product information in end-of-life decision making. Control engineering practice, 15(11):1348-1363.

[Russakovsky et al. 2015] Russakovsky, O., Deng, J., Su, H., Krause, J., Satheesh, S., Ma, S., Huang, Z., Karpathy, A., Khosla, A., Bernstein, M., et al. (2015). Imagenet large scale visual recognition challenge. International Journal of Computer Vision, 115(3):211-252.

[Simonyan and Zisserman 2014] Simonyan, K. and Zisserman, A. (2014). Very deep convolutional networks for large-scale image recognition. arXiv preprint arXiv:1409.1556.

[Sinha and Couderc 2012] Sinha, A. and Couderc, P. (2012). Using owl ontologies for selective waste sorting and recycling. In OWLED-2012.

[Swedberg 2008] Swedberg, C. (2008). Rfid helps reward consumers for recycling. RFID Journal, February.

[Thomas 2008] Thomas, V. M. (2008). Environmental implications of rfid. In Electronics and the Environment, 2008. ISEE 2008. IEEE International Symposium on, pages 1-5. IEEE.

[Yang and Thung ] Yang, M. and Thung, G. Classification of trash for recyclability status. 